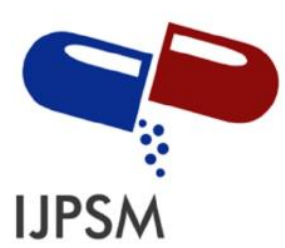

Naserddine Hamadi et al, Int. Journal of Pharmaceutical Sciences and Medicine (IJPSM), Pharma Middle East Virtual Congress (Dec 07-08, 2020)

www.pharma-middleeast.plenareno.com

ISSN: 2519-9889

Impact Factor: 3.426

\title{
Effects of Long-Term Dehydration on Growth Factors, Oxidative Stress and Neuroinflammation in the Hippocampus of the Arabian Camel (Camelus dromedarius)
}

\author{
Naserddine Hamadi' ${ }^{1}$ Ali Mahmoud Hag ${ }^{2}$, Naheed Amir ${ }^{2}$, Hassen Abu Damir ${ }^{2}$ and \\ Abdu Adem ${ }^{3}$
}

\begin{abstract}
${ }^{1}$ Department of Life and Environmental Sciences, College of Natural and Health Sciences, Zayed University, United Arab Emirates
${ }^{2}$ Department of Pharmacology, College of Medicine and Health Science, United Arab Emirates University, United Arab Emirates
\end{abstract} ${ }^{3}$ Department of Pharmacology, College of Medicine \& Health Sciences, Khalifa University, United Arab Emirates

DOI: 10.47760/ijpsm.2020.v05i12.003

\begin{abstract}
The Camels can tolerate loss of water corresponding to $30 \%$ of their body weight, whereas other mammals die from circulatory failure when water loss reaches $12 \%$ of their body weight. We investigated the effect of severe dehydration on microglia, astroglia, and growth factors in the hippocampus.

Camels were divided into three groups; Control $(n=5)$, Dehydrated $(n=8)$ and Rehydrated $(n=6)$. All animals were kept in a shaded corral during the hot summer. All camels were allowed to feed on dry grass ad libitum and water was supplied only to the control group, while the dehydrated and rehydrated camels were not allowed to access water for 20 days. On day 21, Six dehydrated camels were allowed free access to water for $72 \mathrm{hrs}$. At the end of experiment all camels were slaughtered, and the hippocampi were collected for total protein extraction. Astrocytes (GFAP), microglia(IBA-1) and growth factors (IGF-1, NGF- $\beta$ ) were measured by ELISA. In addition, oxidative stress markers SOD, CAT, GSH and MDA were evaluated using colorimetric assay.

Our results showed a significant increase in the levels GFAP and IBA-1 compared to the controls, while rehydration significantly normalized their levels in the hippocampus. IGF1 and NGF- $\beta$ levels were significantly increased in the hippocampus after dehydration, whereas the rehydration failed to bring the levels of these growth factors to the control levels. Surprisingly, our results showed no effect of dehydration on the oxidative markers (SOD, CAT, GSH and MDA) in the hippocampus in dehydrated and control groups.

In conclusion, the current study showed clearly after long term dehydration a state of microgliosis and astrocytosis in the hippocampus accompanied by depletion in key growth factors, IGF-1 and NGF- $\beta$. In addition, no effect on oxidative stress was observed in the hippocampus following long term dehydration.
\end{abstract}

